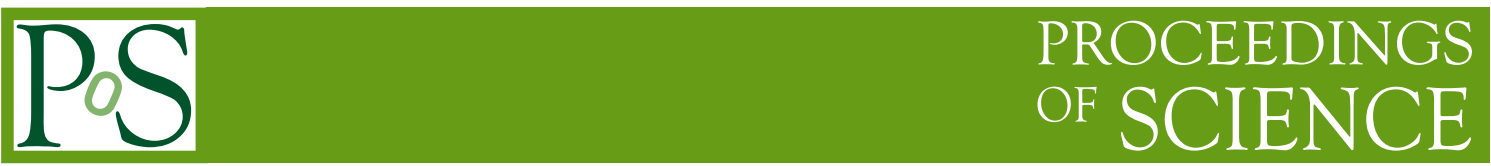

\title{
ESA Gaia and microquasars
}

\author{
René Hudec \\ Astronomical Institute of the Academy of Sciences of the Czech Republic \\ E-mail: rene.hudec@gmail.com
}

\section{Vojtěch Šimon}

Astronomical Institute of the Academy of Sciences of the Czech Republic

E-mail: vojtech.simon@gmail.com

\section{Lukáš Hudec}

Astronomical Institute of the Academy of Sciences of the Czech Republic

E-mail: matfyzak@seznam.com

\begin{abstract}
The ESA satellite in development Gaia to be launched in 2011 will focus on highly precise astrometry of stars and all objects down to limiting magnitude 20. Albeit focusing on astrometry related matters, the satellite will also provide photometric and spectral information and hence important inputs for various branches of astrophysics. Within the Gaia Variability Unit CU7 and related work package Specific Object Studies there has been a sub-work package accepted for optical counterparts to celestial high-energy sources, a category which includes the optical counterparts (i.e. optical transients and optical afterglows, including counterparts of XRFs and yet hypothetical orphan afterglows) of GRBs, and also microquasars. Although the sampling of photometric data will not be optimal for this type of work, the strength of Gaia in such analyzes is the fine spectral resolution (spectro-photometry), which will allow the correct classification of related triggers. The possibilities to detect and to analyze optical transients and optical afterglows of GRBs and microquasars by Gaia will be presented and discussed.
\end{abstract}

VII Microquasar Workshop: Microquasars and Beyond

September 1 - 5, 2008

Foca, Izmir, Turkey 


\section{Introduction}

Gaia is a cornerstone astrophysical mission of the European Space Agency ESA. It is a global space astrometry mission (Perryman, 2005). Its goal is to make the largest, most precise map of our Galaxy by surveying an unprecedented number of stars. Gaia is a mission that will conduct a census of billions stars in our Galaxy. It will monitor each of its target sources about 100 times over a five-year period. It is expected to discover hundreds of thousands of new celestial objects, such as extra-solar planets and failed stars called brown dwarfs. Within our own Solar System, Gaia should also identify tens of thousands of asteroids. Gaia will measure the positions, distances, space motions, and many physical characteristics of some one billion stars in our Galaxy and beyond (see http://astro.estec.esa.nl for more details).

\section{Optical counterparts of high-energy sources}

It is obvious that, with the above briefly described performance, Gaia will provide valuable inputs to various research fields of contemporaneous astronomy and astrophysics including the field of high-energy sources. Most of the variable object research will be performed within the Gaia Variability Coordination Unit CU7. To study the optical counterparts of celestial high-energy sources, there will be several advantages provided by Gaia. First, this will be a deep limiting magnitude of 20 (Jordi and Carrasco, 2007), much deeper than most of previous studies and global surveys. For example, no detailed statistics of variable stars has been investigated for magnitudes fainter than 18. Secondly, the time period covered by Gaia observations, i.e. 5 years, will also allow some studies requiring long-term monitoring, recently provided mostly by astronomical plate archives and small or magnitude-limited sky CCD surveys. But perhaps the most important benefit of Gaia for these studies will surely be the color (spectral) resolution thanks to the low-resolution (prism) Gaia spectroscope. This will allow some detailed studies involving analysis of the color and spectral changes not possible before. Another valuable input will come from the parallax measurements the knowledge of directly measured distances of the sources will be highly beneficial. The details of studies of the optical counterparts of high-energy sources have been recently evaluated and are described in more detail mostly by the dedicated sub-workpackages within the workpackage Specific objects studies within the Gaia CU7 (Hudec et al., 2007a, Hudec et al., 2007b).

The main objective of the sub-workpackage mentioned above is the investigation and analysis of the optical counterparts of high-energy astrophysical sources (including High-Mass X-Ray Binaries, Low-Mass X-Ray Binaries, X-Ray Transients, X-Ray Novae, Optical Transients and Optical Afterglows related to X-Ray Flashes and Gamma-Ray Bursts, Microquasars etc.) based on the Gaia data as complex analysis with additional data.

\section{The high energy sources perspectives}

In this section we briefly list and discuss the main types of the cosmic high-energy sources whose optical counterparts are expected to be investigated by Gaia.

High-Mass X-Ray Binaries (HMXBs) - systems consisting of a compact, mass accreting object (neutron star (NS) or a black hole (BH), rarely also a white dwarf (WD)) and an early-type 
star (O, B) donating mass (donor). Basic modes of the mass transfer are: (a) Roche lobe overflow; (b) radial stellar wind in case of supergiant donors; (c) circumstellar disk in case of a Be main sequence star. Periastron passage of the compact object leads to a periodic variability in some HMXBs, especially if they contain a Be component and have an eccentric orbit (see also below). The optical luminosity of HMXBs is usually dominated by the donor star, not by the matter flowing onto the compact object. The usual kinds of the optical activity are the orbital modulation and longterm fluctuations, only rarely outbursts. About a hundred HMXBs have been discovered in X-rays in our Galaxy but it is very probable that more of them are hidden because they are in a low X-ray state or their X-ray emission is highly absorbed.

Low-Mass X-Ray Binaries (LMXBs) - systems consisting of a compact object (NS or BH) and a Roche lobe filling companion star donating mass. The spectral type of the donor and the orbital period are related. It is usually a late type main-sequence star, but also rare cases where it is a subgiant, giant or a WD are known (depending on the orbital period ranging from about $11 \mathrm{~min}$ to several days (rarely months), mostly several hours). The optical luminosity is often dominated or at least largely influenced by the matter flowing onto the compact object. LMXBs are often highly variable on various time scales, from seconds to decades (see Fig.2). They display various kinds of activity, like high/low state transitions (e.g. HZ Her/Her X-1, see Fig.1) or outbursts (see below). Some of them also display the orbital modulation caused by irradiation of the donor (e.g. HZ Her) or eclipses of the accretion disk by the donor.

X-ray transients (XTs) - a subtype of HMXBs and LMXBs. They are characteristic by their transient increase of X-ray flux, often by several orders of magnitude. Some XTs in quiescence may not be detectable in X-rays by the current instruments. In the optical, the quiescent luminosity may be dominated by the donor star even in the case of a LMXB.

XTs can be sorted according to the physical processes:

(a) Soft X-ray transients ( $S X T$ s), also called X-ray novae, are caused by the thermal instability of the accretion disk in a LMXB. The physical process is analogous to that in dwarf novae, but is more energetic and irradiation of the disk by X-rays from the close vicinity of the compact object plays a large role during outburst. Both X-ray and optical outburst is observed (e.g. Aql X1/V1333 Aql, XTE J1118+480/KV UMa). The optical luminosity rises by several magnitudes. The duration of the outburst is weeks to months, while the recurrence time (i.e. interval between outbursts) is months to decades.

(b) Hard X-ray transients - (i) Type I outbursts are dependent on the orbital period. They occur during periastron passage of the compact object and are attributed to an increase of the mass accretion from the Be donor. This mechanism suggests periodicity but these outbursts may not occur during every passage. The reason is that the amount of the circumstellar matter originated from the $\mathrm{Be}$ donor undergoes long-term variations. Usually the increase of the optical flux in the continuum is quite small during the X-ray outburst, but there are some important exceptions: A0538-66 in LMC displays the optical outbursts with an amplitude of about 2 mag with the period of 16.5 days. (ii) Type II outbursts are independent on the orbital phase, i.e. on the position of the compact object on its orbit. They are probably caused by a dramatic expansion of the circumstellar disk.

(c) Very fast X-ray transients - quite new kind of transient, only a few are known yet. Each outburst, yet observed only in X-rays, lasts for at most several hours, but they can be relatively 
frequent (the recurrence time is of days in some cases). They seem to occur in some HMXBs but the outburst mechanism is uncertain. The optical activity of the source is unknown, especially it is not known if the X-ray outburst is accompanied by any optical brightening. Also the general optical activity (brightness and color changes) including long-term variations is not known because these objects were only recently identified.

Microquasars - X-ray binaries which possess relativistic jets, i.e. collimated outflows of matter moving at relativistic velocities from the compact component (e.g. V4641 Sgr). Some of them display large-amplitude outbursts in various spectral passbands, from far X-rays through optical to radio.

Optical transients (OTs) and Optical afterglows (OAs) related to X-ray flashes (XRFs) and gamma-ray bursts (GRBs) - GRBs are the most energetic events in the Universe. They are caused either by a core-collapse of a very massive star or a collision of two compact objects. The GRB event lasts typically from seconds to minutes, but is often followed by an OA which lasts for days or even longer. Typically, OAs reach the optical magnitude around 18 in the initial phase, but some are brighter. The specific spectral profile of many OAs gives us a good hope to identify them among transients on the basis of even a single spectrophotometric observation with Gaia. Furthermore, it will be possible to investigate the field observed with Gaia where a GRB was recently observed by a gamma-ray satellite like e.g. Swift and search for a possible optical transient (even a search for delayed transients can be made). The long-lasting coverage of the sky with the Gaia instruments also gives us a hope to search for the so-called orphan afterglows. These objects are GRBs from which no gamma-ray emission was detected (despite it is emitted) because of a different beaming of the high-energy and optical emission - they are predicted by theory. In addition, the Gaia Science Alerts are expected to be generated at least for some of the strong cases of OAs observed by Gaia, mostly in the cases when the fading OA will be covered by several following transits of the object in the focal plane.

The relatively dense coverage of the light curves especially of X-ray binaries in the optical passband can be achieved with the ground-based monitoring telescopes, like those operated by the Ondřejov group (e.g. BART).

The great and unique power of the Gaia mission will be the study of the photometric properties of the optical counterparts of high-energy sources, in particular, in a large number of filters (spectrophotometry). In co-operation with the ground-based instruments, the state of the activity of a given object observed by Gaia can be identified in many cases. The Gaia data will enable us to create detailed color-color and color-magnitude diagrams and analyze the position of an optical counterpart of a given X-ray source in a given state of its activity. Also shifts of a given object in such diagrams during various states of its activity, as well as population studies will be of a great merit.

Specifically we aim: (1) For selected targets, multispectral analysis using Gaia and other databases (such as the X-ray and gamma-ray satellite data, optical ground-based data etc.) may be feasible, (2) analysis of long-term light changes and their evolution, (3) analysis of active states, outbursts and flares, (4) the study and understanding of related physical processes, (5) spectrophotometry, relation of brightness and spectrum/color, (6) for selected sources, dedicated complex analysis, and (7) statistics of the whole sample of objects.

Below we briefly list and discuss the suggested methods, and their results, proposed to achieve 


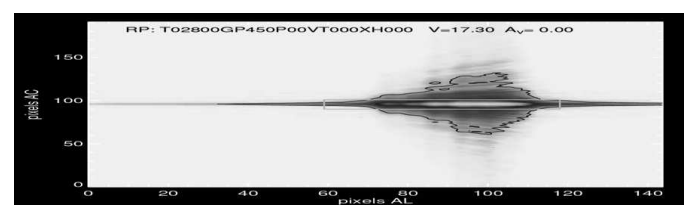

Figure 1: The simulated Gaia low-dispersion spectrum (left) and a real low-dispersion spectrum from digitized Schmidt astronomical Sonneberg Observatory plate (right).

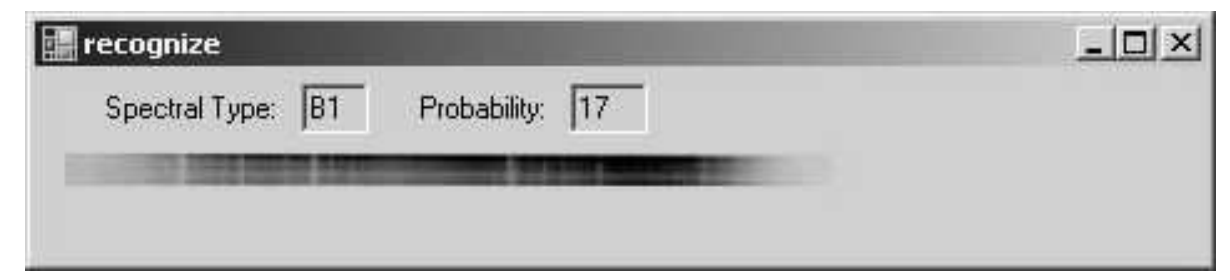

Figure 2: The computed classification details of particular star image on Schmidt spectral plate taken with objective prism.

the objectives described above. More details are given in Hudec and Simon, 2007ab. (1) Use of the tools of standard time-series analysis (Fourier and wavelet analysis, methods of statistical timeseries analysis (correlation, autocorrelation, noise and signal detection etc.), deterministic/chaotic behavior) to reveal the physical mechanisms of variations, (2) Study of the flaring behavior of objects including spectral changes (color-color diagrams, color evolution), study of the correlation between the brightness changes and spectral (color) variations, statistical analysis of the flaring behavior of the whole data sample, (3) Study of activity/inactivity modes incl. spectral changes, physical classification, and (4) Complex analysis with Gaia and supplementary data including data from dedicated ground-based robotic telescopes as well as from other sources (inc. satellite data).

\section{The spectral power}

The Gaia telescopes offer unique variability studies based on low-dispersion spectra, i.e. the energy resolution of recorded star images (as these are represented by prism low-dispersion spectra). In this context, the application of algorithms developed for digitized astronomical archival plates (Hudec, 2007) may be important for Gaia (Fig.3). The novel algorithms for automated analysis of digitized spectral plates have been recently developed by informatics students (Hudec, 2007) and are suitable for (1) Automated classification of spectral classes, (2) Searches for spectral variability (both continuum and lines), (3) Searches for objects with specific spectra, (4) Correlation of spectral and light changes, and (5) Searches for transients.

The archival spectral plates taken with the objective prism offer the possibility to simulate the Gaia low-dispersion spectra and related procedures such as searches for spectral variability and variability analysis based on spectrophotometry (low dispersion prism spectra). In recent development, we focus on sets of spectral plates of the same sky region covering long time intervals with good sampling. 


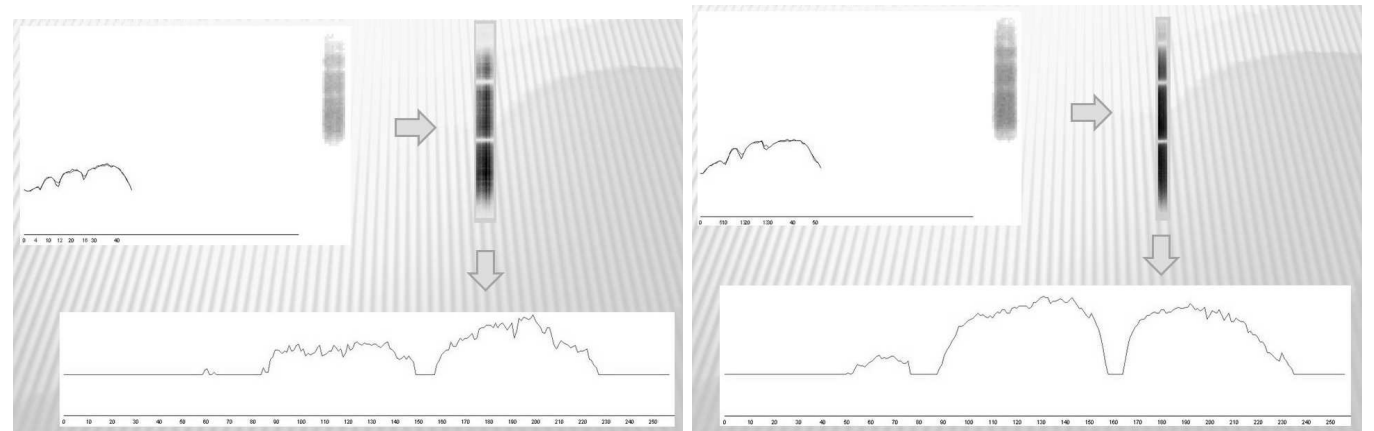

Figure 3: Two examples of automated spectral classification of stars with low-dispersion spectra according to Hudec, 2007. In each image, upper left is the star spectrum, and bottom right the automatically found model (atlas) spectrum.

\section{Gaia and Microquasars}

\subsection{Photometry}

From the 15 confirmed microquasars in our Galaxy, 10 are within the Gaia limit on mag 19 (and 5 are even brighter than mag 15), hence their long-term light curves will be obtained with high accuracy. However, the sampling provided by Gaia is not optimal for goals of detection of new microquasars, hence not always we can expect reliable and confirmed detection of microquasars based only on photometry by Gaia

\subsection{Spectroscopy}

The primary strength of Gaia for the microquasars study is the fine spectro-photometry and low-dispersion spectroscopy. This can give additional, so far little exploited additional input to the study and investigation of microblazars, and perhaps even to help search for and to verify additional galactic microquasars

\subsection{Colors of Microquasars}

The color indices are characteristic for each object, since the scatter of the colors in the diagram is larger than that introduced by the shift of a given object in the diagram.

The available microquasars (LMXBs in outburst, HMXBs) in the $(U-B)_{0}$ vs. $(B-V)_{0}$ diagram, display blue colors, with a trend of a diagonal formed by the individual objects. Their color indices corrected for the interstellar reddening are confined to $-1.4<(U-B)_{0}<-0.6$, $-0.4<(B-V)_{0}<0.4$. This characteristic can be used as a basic diagnostic method to pick up potential microquasars in the huge data sets from the Gaia satellite. This method can be used even for the optically faint, hence distant objects.

The method of the color indices yields valuable results already in the studies of other HE sources, e.g. OAs of GRBs. The fine color resolution of Gaia, together with long-term monitoring, will contribute to the understanding of microquasars considerably. 


\section{Conclusion}

The Gaia mission of European Space Agency ESA will contribute essentially to scientific studies and physical understanding of the optical counterparts of high-energy sources. The variability studies of faint objects based on the optical low-resolution spectra are expected to provide unique novel data and may use the algorithms recently developed for automatic analysis of digitized spectral Schmidt plates.

\section{Acknowledgments}

The Czech participation in the ESA Gaia project is supported by the PECS project 98058 . The scientific part of the study is related to the grant 205/08/1207 provided by the Grant Agency of the Czech Republic. Some aspects of the project described here represent a natural continuation of the Czech participation to the ESA INTEGRAL (ESA PECS 98023).

\section{References}

[1] M. A. C. Perryman, in Astrometry in the Age of the Next Generation of Large Telescopes, ASP Conference Series, Vol. 338, San Francisco: Astronomical Society of the Pacific, p.3 (2005).

[2] C. Jordi, J. M. Carrasco, in The Future of Photometric, Spectrophotometric and Polarimetric Standardization, ASP Conference Series, Vol. 364, San Francisco: Astronomical Society of the Pacific, p.215 (2007).

[3] R. Hudec, W. Wenzel, Observations of HZ Her/Her X-1 on Sonneberg Astrograph Plates, BAICz 27, 325 (1976).

[4] R. Hudec, Optical Properties of X-ray Stars. 2. The Secular Brightness Variations of the X-ray System V 818 Sco/Sco X-1, BAICz 32, 108 (1981).

[5] R. Hudec, V. Simon, 2007a, Specific object studies for cataclysmic variables and related objects ESA Gaia Reference Code GAIA-C7-TN-AIO-RH-001-1.

[6] R. Hudec, V. Simon, 2007b, Specific object studies for optical counterparts of high energy sources. ESA Gaia Reference Code GAIA-C7-TN-AIO-RH-002-1.

[7] L. Hudec, Algorithms for spectral classification of stars, BSc. Thesis, Charles University, Prague (2007). 